\title{
Evaluation of maternal and newborn health services in Jordan
}

This article was published in the following Dove Press journal: Journal of Multidisciplinary Healthcare

\section{Yousef S Khader' Mohammad S Alyahya ${ }^{2}$ Nihaya A Al-Sheyab ${ }^{3}$ Khulood K Shattnawi ${ }^{4}$ Hind Rajeh Saqer ${ }^{5}$ Anwar Batieha ${ }^{6}$ 'Epidemiology, Medical Education and Biostatistics, Department of Community Medicine, Public Health and Family Medicine, Faculty of Medicine, Jordan University of Science and Technology, Irbid, Jordan; ${ }^{2}$ Department of Health Management and Policy, Faculty of Medicine, Jordan University of Science and Technology, Irbid, Jordan; ${ }^{3} \mathrm{Child}$ and Adolescent Health, Allied Medical Sciences Department, Faculty of Applied Medical Sciences, Faculty of Nursing, Jordan University of Science and Technology, Irbid, Jordan; ${ }^{4}$ Maternal and Child Health Nursing Department, Faculty of Nursing/WHO Collaborating Center, Jordan University of Science and Technology, Irbid, Jordan; ${ }^{5}$ Faculty of Medicine, Jordan University of Science and Technology, Irbid, Jordan; ${ }^{6}$ Epidemiology Department of Community Medicine, Public Health and Family, Faculty of Medicine, Jordan University of Science and Technology, Irbid, Jordan}

Correspondence: Yousef S Khader Epidemiology, Medical Education and Biostatistics, Department of Community Medicine, Public Health and Family Medicine, Faculty of Medicine, Jordan University of Science and Technology, Irbid 221 10, Jordan

Tel +962796802040

Fax+962 27201064

Email yskhader@just.edu.jo
Objectives: This study aimed to assess the maternal and newborn services in Jordanian hospitals to provide policymakers, health professionals, and researchers with a clear picture about the current status of maternal and newborn health services.

Methods: A total of 32 main hospitals that provide maternity services in Jordan were assessed. The study involved direct observations of these hospitals and interviews with basic health and hospital staff, with the purpose of assessing and evaluating the availability of various services for mothers and newborns, availability of resources, equipment and supplies, documentation and staff training, and provision of the health care services.

Results: Some hospitals had shortages of obstetricians and gynecologists, pediatricians, neonatologists, and midwives/nurses. Antenatal care was not provided systematically in many hospitals across the country. A lack of necessary equipment, drugs, and supplies was evident in some hospitals. Admission departments of some hospitals had insufficient supplies. The operation theaters in many hospitals lacked a variety of necessary equipment including some basic items such as thermometers and some advanced items such as resuscitation sets for babies. Only two-thirds of all delivery rooms in the selected hospitals had radiant heaters and obstetrical stethoscopes available. A significant lack of neonatal ICU equipment was found such as incubators, resuscitation tables, continuous positive airway pressure, $\mathrm{O} 2$ oximeters, and phototherapy.

Conclusion: The findings revealed an overall satisfactory quality of maternal and newborn care and services, however, some deficiencies existed. The findings are expected to aid policymakers, health professionals, and researchers to recognize the gaps in the processes, supplies, and quality of care related to the provided services at maternal facilities and help them to design and implement evidence-based health programs in order to provide effective health services and promote the health of mothers and newborns.

Keywords: maternal and newborn health services, assessment, quality of care, Jordan

\section{Introduction}

Quality of health care assessment is the process of evaluating the performance of health care providers and health plans according to the recognized quality standards., ${ }^{1,2}$ Recently, the WHO published a "framework" for improving the quality of care for mothers and newborns around the time of childbirth. This framework, which was published in 2016, contains domains of quality of care that should be assessed, improved, and monitored within the health facility. Based on this framework, six strategic areas were identified to improve the quality of maternal and newborn care. The six areas are clinical guidelines, standards of care, effective interventions, measures of quality of care, relevant research, and capacity-building. ${ }^{3}$ 
The maternal mortality ratio (MMR), which is an important indicator of the quality of health care in any country, has fallen globally by $44 \%$ since $1990,{ }^{4}$ a progress which is substantial but not sufficient enough to achieve the millennium development goal (MDG) 5 target of a three-quarter drop. Maternity care in Jordan is generally of a high standard. The majority (94\%) of Jordanian women have at least four antenatal visits and about $98 \%$ of mothers are delivered within a health institution providing skilled care at birth., Jordan conducted its national maternal mortality study in 2007-2008. The MMR was reported at 19.1 per 100,000 live births (LB). ${ }^{6}$ The study highlighted clearly that a high percentage of maternal deaths were avoidable with a high contribution of remediable factors, and about half of deaths were assessed to having some degree of suboptimal care. On the other hand, infant and under-five mortality rates in Jordan have decreased steadily over the last two decades, reaching 21 and 24 per 1,000 LB, respectively, in 2007. 7-9 Despite such significant improvement, babies are still at risk during the perinatal period, which covers pregnancy, delivery, and the postpartum period, as well as during the neonatal stage. In the late 1990s, the neonatal mortality rate (NNMR) in Jordan fell from 19 to 15 per 1,000 LB and remained relatively constant as Jordan transitioned into the new millennium. ${ }^{10}$

To achieve the Sustainable Development goals ${ }^{11}$ and to achieve the goals and targets of the "Every Newborn: an action plan to end preventable deaths," ${ }^{\prime 2}$ particular focus must be placed on reducing neonatal mortality. This will require effective planning and monitoring of health services and, most importantly, accurate measures of mortality.

The Jordan Perinatal and Neonatal Mortality Study (JPNM), initiated and funded by UNICEF/Jordan, was an effort to gather reliable neonatal and perinatal mortality data. ${ }^{13}$ Using the gestational age cutoff value of $\geq 20$ weeks, stillbirth, neonatal, and perinatal mortality rates were $11.6 / 1,000$ total births (TB), 14.9/1,000 LB, and 23.7/1,000 TB, respectively. Using the Ministry of Health's (MoH) Clinical Practice Guidelines for Care of the Newborn to assess the appropriateness or optimality of the care, the JPNM study experts' panel determined that only $37.3 \%$ of newborns who died during the neonatal period had received optimal medical care. ${ }^{13}$

Research has shown that securing access to skilled, competent health care professionals as well as comprehensive, appropriate care around delivery can substantially minimize maternal and newborn mortality and morbidity, ${ }^{14}$ including preventable causes of maternal and newborn deaths. ${ }^{15,16}$ Research has also highlighted that high-quality care requires accurate use of the available infrastructure, staff, and commodities to ensure effective case management. ${ }^{17}$ Therefore, improvement of the quality of preventive and curative care during this critical period could have a great impact on maternal, fetal, and newborn survival. Assessing the current status of the quality of care in providing maternal and newborn health care according to recognized quality standards can pinpoint the areas that need to be improved to reach optimal care for both the mother and her fetus/newborn. This study aimed to assess the maternal and newborn services in Jordanian hospitals and to provide policymakers, health professionals, and researchers with a clear picture about the current status of maternal and newborn health services and thus enable them to design and implement evidence-based health programs to provide effective health services and promote the health of mothers and newborns.

\section{Methods}

\section{Selection of maternities}

A total of 32 maternity hospitals were selected from a total of 103 hospitals in Jordan; 11 hospitals in the Middle region, 14 hospitals in the North region, and seven hospitals in the South region. They represent the maternity hospitals in 12 governorates of Jordan and in different health sectors, namely, the MoH, the Royal Medical Services (RMSs), the private sector, and the university hospitals. The hospitals were selected based on the following criteria:

1. Representation of the three regions of Jordan, namely, the South, Middle, and North;

2. Representation, within the three regions, of the three health sectors, $\mathrm{MoH}$, private, and military;

3. Inclusion of at least one university hospital; and

4. Consideration of the workload of the hospitals (number of deliveries) as well as the geographic distribution, which is likely to be related to the socioeconomic status of clients and the quality of services provided.

The difference in representation from each region is related to the size of the region. Ethical approvals were obtained from the Intuitional Review Board (IRB) at Jordan University of Science and Technology and from the IRB at Jordan Ministry of Health. The official approval for direct observation was obtained from the hospitals' directors, and all interviewed staff had signed the consent form.

\section{Study instruments}

The maternal and neonatal health services in the selected hospitals were assessed to evaluate the availability of various 
services for mothers and newborns, availability of resources, equipment and supplies, documentation and staff training, and provision of the health care services. The assessment took place during July-October 2017. A total of five experts were recruited and trained to assess the maternal and neonatal services in the selected hospitals. Three experts (one obstetrician, one neonatologist, and one senior midwife) were recruited from Jordan $\mathrm{MoH}$ staff and two experts in the area of Maternal and Child Health were recruited from the University staff. The assessors received training for 2 days to develop a common understanding of the purpose and the objectives of facility assessment, to have input on the assessment forms, and to understand and be able to use the assessment forms. The training familiarized data collectors with the forms and provided instructions needed to complete the forms. The assessment forms (Supplementary material S1) were developed to collect information in all key areas that have a major impact on neonatal outcomes, identify the areas where poor or substandard care is provided, and involve managers and staff at facility level in identifying actions to improve quality of care. All assessment and interview forms were pilot tested in three hospitals and modified based on the feedback of the hospitals' staff.

In general, the forms were structured as standard-based checklists and question/answer format covering important main areas: general hospital infrastructure and services, maternity ward/nursery and neonatal ward, routine neonatal care, sick newborn care, infection prevention and supportive care, human resources, and essential safety and life-saving practices. The assessment forms utilized different sources of information including direct observation and semi-structured interviews with staff. The forms included indicators and questions for assessing newborn care services at facilities and were developed based on the WHO tool, a tool developed by the Inter-agency Newborn Indicators Technical Working Group and the service provision assessment (SPA) tool developed by ICF International under the USAID-funded MEASURE DHS project. ${ }^{18,19}$ The tool aimed to collect data on indicators that assess whether a facility is able to address the three major causes of newborn deaths - intrapartum causes (birth asphyxia), preterm births, and infection. Indicators are divided into the following categories: service availability, equipment and supplies, documentation, trained staff, supervision, and additional optional indicators

\section{Data collection and analysis}

The data collection team was responsible for maintaining a $\log$ of all the facilities visited, the dates of the visits, and any important comments about the visits. Prior to visits, the health facility was informed about the time and date of the visit of the assessment team. Upon arrival at the facility, the team met the facility director or another officer in charge, explained the purpose of the visit, introduced the data collection team, presented the study authorization letter, and received permission to begin data collection. The data collectors recorded data according to the instructions offered during training by asking questions, listening, and observing. Their role was not to teach, critique, or advise. The service delivery was not interrupted during the visits. The data collection team was careful not to compromise provider-patient confidentiality. The duration of assessment varied from 3 to 5 hours depending on the size of the hospital. The assessors met the day after the visit to discuss findings. All filled questionnaires received from the assessors were edited and coded before data entry.

Data were entered into IBM SPSS version 20 by one of the assessors. Data entry was checked and cleaned by the principal investigator. Data were analyzed using IBM SPSS and presented as overall estimates and percentages and by region.

\section{Results}

\section{Hospitals' characteristics}

A total of 32 hospitals distributed over the three regions of the country (14 in the north, 11 in the middle, and seven in the south) and covering different sectors (17 MoH hospitals, five RMS hospitals, nine private hospitals and one University hospital) were assessed. Princess Badea Hospital and Princess Rahma Hospital were assessed as one unit because of proximity and overlapping of staff and services. All hospitals in all regions had a ward for admitting obstetrics patients, a theater for performing cesarean section (CS), and a neonatal intensive care unit (NICU). The number of beds for admitting obstetric patients ranged from 3 to 98 and the number of beds in the NICU ranged from 3 to 65 . Three hospitals $(9.4 \%)$ had no separate room or ward for admitting "infectious" cases.

\section{Human resources}

All hospitals had a person (specialist/resident) skilled in performing deliveries at all times ( 24 hours a day), including weekends, to provide delivery care. Of those, a skilled specialist/resident is present at all times in $22(68.8 \%)$ hospitals and is on call in $10(31.3 \%)$ hospitals. As perceived by health professionals in the studied hospitals, the number of $\mathrm{Ob} \&$ Gyns was inadequate in $10(31.3 \%)$ hospitals, the number of pediatricians was inadequate in $10(31.3 \%)$ hospitals, 
the number of neonatologists was inadequate in $14(43.8 \%)$ hospitals, the number of midwives/nurses providing obstetric care was inadequate in $12(37.5 \%)$ hospitals, and the number of pediatric nurses was inadequate in 20 (64.5\%) hospitals.

$\mathrm{MoH}$ hospitals were more stressed by the shortage of human resources, especially of neonatologists and pediatric nurses. The majority of $\mathrm{MoH}$ hospitals suffered from shortages in the number of neonatologists and pediatric nurses. The university hospital had an inadequate number of neonatologists and pediatric nurses and five private hospitals out of nine had an inadequate number of neonatologists. The shortage was evident in RMSs for midwives/nurses (obstetrics) and pediatric nurses

\section{Basic and comprehensive emergency obstetric care (CEmOC) and neonatal care}

Almost all hospitals offer the basic and CEmOC and neonatal care, including normal vaginal delivery, CS, administration of parenteral antibiotics or anticonvulsants, manual removal of the placenta, removal of retained products after delivery, basic neonatal resuscitation (eg, with a bag and a mask), and intubation/ventilation for baby (Table 1). Corticosteroids for premature labor were not available in one hospital in the north. This same hospital does not perform assisted vaginal delivery (eg, vacuum extraction, forceps delivery). Four hospitals (two $\mathrm{MoH}$ hospitals and two private hospitals) do not perform continuous positive airway pressure (CPAP) for baby.

\section{Antenatal care}

All hospitals were found to provide antenatal care except one private hospital. Table 2 shows the number of hospitals that do not provide selected services during pregnancy or provide them but not systematically. Services such as blood pressure and hemoglobin measurements were available and were provided systematically in all hospitals. However, other services including iron supplementation, folic acid supplementation, fasting blood sugar measurement, urine stick (protein, sugar), tetanus toxoid (TT) vaccination, breastfeeding counseling, and family planning counseling were not fully implemented during pregnancy and were not provided systematically in some hospitals. TT vaccination was not provided systematically or not provided at all in almost all hospitals in the north, $63.6 \%$ of hospitals in the middle, and all hospitals in the south. In about half of the hospitals of the three regions, iron supplementation was not systematically provided. These services were found to be more commonly provided in the $\mathrm{MoH}$ hospitals compared to RMSs and private hospitals. All

Table I The availability of basic and comprehensive emergency obstetric care and neonatal care services in the selected hospitals ${ }^{\mathrm{a}}$

\begin{tabular}{|c|c|c|c|c|c|c|c|c|}
\hline \multirow[t]{2}{*}{ Type of service } & \multicolumn{2}{|c|}{ North $N=14$} & \multicolumn{2}{|c|}{ Middle $\mathbf{N}=$ I I } & \multicolumn{2}{|c|}{ South $\mathbf{N}=7$} & \multicolumn{2}{|c|}{ Total $\mathbf{N}=32$} \\
\hline & $\mathbf{n}$ & $\%$ & $\mathbf{n}$ & $\%$ & $\mathbf{n}$ & $\%$ & $\mathbf{n}$ & $\%$ \\
\hline Administration of uterotonic drugs (ie, parenteral oxytocin) & 14 & 100.0 & II & 100.0 & 6 & 85.7 & 31 & 96.9 \\
\hline Perform assisted vaginal delivery (eg, vacuum extraction, forceps delivery) & 13 & 92.9 & II & 100.0 & 7 & 100.0 & 31 & 96.9 \\
\hline Corticosteroids for preterm labor & 13 & 92.9 & II & 100.0 & 7 & 100.0 & 31 & 96.9 \\
\hline CPAP for baby & II & 78.6 & 10 & 90.9 & 7 & 100.0 & 28 & 87.5 \\
\hline
\end{tabular}

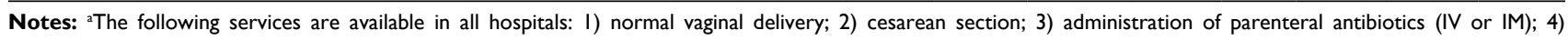
administration of parenteral anticonvulsants for preeclampsia and eclampsia (ie, magnesium sulphate); 5) manual removal of the placenta; 6) removal of retained products after delivery (eg, manual vacuum aspiration); 7) basic neonatal resuscitation (eg, with bag and mask); 8) intubation/ventilation for baby; and 9) blood transfusion.

Abbreviation: CPAP, Continuous positive airway pressure.

Table 2 The number of hospitals that do not provide selected services during pregnancy or provide them but not systematically ${ }^{a}$

\begin{tabular}{|c|c|c|c|c|c|c|c|c|}
\hline \multirow[t]{3}{*}{ Services } & \multicolumn{8}{|c|}{ Not provided or provided but not systematically } \\
\hline & \multicolumn{2}{|c|}{ North $\mathbf{N}=14$} & \multicolumn{2}{|c|}{ Middle $\mathbf{N}=$ I I } & \multicolumn{2}{|c|}{ South $\mathbf{N}=7$} & \multicolumn{2}{|c|}{ Total $\mathbf{N}=32$} \\
\hline & $\mathbf{n}$ & $\%$ & $\mathbf{n}$ & $\%$ & $\mathbf{n}$ & $\%$ & $\mathbf{n}$ & $\%$ \\
\hline Iron supplementation & 9 & 64.3 & 5 & 45.5 & 3 & 42.9 & 17 & 53.1 \\
\hline Folic acid supplementation & 6 & 42.9 & 4 & 36.4 & 0 & 0.0 & 10 & 31.3 \\
\hline Fasting blood sugar & 9 & 64.3 & 3 & 27.3 & 4 & 57.1 & 16 & 50.0 \\
\hline Urine stick (protein, sugar) & 4 & 28.6 & $\mathrm{I}$ & 9.1 & 0 & 0.0 & 5 & 15.6 \\
\hline TT vaccination & 13 & 92.9 & 7 & 63.6 & 7 & 100.0 & 27 & 84.4 \\
\hline Breastfeeding counseling & 5 & 35.7 & 2 & 18.2 & I & 14.3 & 8 & 25.0 \\
\hline Family planning counseling & 6 & 42.9 & I & 9.1 & 0 & 0.0 & 7 & 21.9 \\
\hline
\end{tabular}

Notes: ${ }^{a}$ All hospitals offer blood pressure and hemoglobin measurements.

Abbreviation: TT, tetanus toxoid. 
of these services were found to be provided systematically in the university hospital.

\section{Admission department}

The admission departments of a few hospitals, mainly private hospitals in the middle region, had insufficient supplies of inexpensive items such as essential equipment, consumables, and drugs. The admission departments of three hospitals (one private hospital in the north region and two private hospitals in the middle region) lacked an emergency set. Two hospitals had no obstetrical stethoscope. Medications such as magnesium sulfate, diazepam, and oxytocin were missing in five to six hospitals. Posters or information on hand washing were missing in almost one third of hospitals.

\section{Delivery room}

Table 3 shows the availability of safety/lifesaving essential equipment and consumables in the delivery rooms of the 32 hospitals. For delivery services, items and equipment were generally available, but not all items needed to guarantee high quality care were available. The delivery rooms of some hospitals suffered from shortages in essential equipment. Electronic thermometers for measuring babies' temperature were available in all delivery rooms of $10(31.3 \%)$ hospitals only. Moreover, room thermometers were available in all delivery rooms of $10(31.3 \%)$ hospitals only, indicating that room temperature was not monitored in the delivery rooms of 22 hospitals. A radiant heater and obstetrical stethoscope were available in all delivery rooms of twothirds of hospitals.

\section{Delivery block/department}

Emergency equipment necessary for advanced newborn resuscitation in delivery blocks/departments was missing in two hospitals. Delivery rooms of only 12 hospitals had a separate fridge for blood components.

\section{Operation theater (OT)}

OTs of some hospitals lacked necessary equipment. Thermometers were available in all OTs of 15 (46.9\%) hospitals only. A set of advanced resuscitation for babies was available in $22(68.8 \%)$ hospitals. A transportation heated cradle/ incubator for babies was missing in OTs of two-thirds of hospitals.

Only 18 (56.3\%) hospitals had a warming device for the newborn in the OT. Oxygen was not available in the OT of one hospital in the south.

\section{$\mathrm{NICU}$}

NICUs of all hospitals except one had a source of oxygen available for each baby and had a vacuum aspirator in each section. NICUs in some hospitals lacked basic and essential equipment. About one-third of hospitals did not have a resuscitation set in all sections. A breastfeeding room or corner was missing in four hospitals and fridges for storing breast milk were missing in almost half the hospitals. A wall thermometer was present in each section/room of $12(37.5 \%)$ hospitals only and an individual thermometer for each infant was present in $24(75.0 \%)$ hospitals. Infants were seen wearing hats in the incubator/cradle of two hospitals only. Babies sharing the same incubator or cradle (excluding twin, triplet) was seen

Table 3 The availability of essential equipment and consumables in the delivery rooms

\begin{tabular}{|c|c|c|c|c|c|c|c|c|}
\hline \multirow{3}{*}{$\begin{array}{l}\text { Safety/saving life medical } \\
\text { equipment/supply }\end{array}$} & \multicolumn{8}{|c|}{ Availability in all delivery rooms } \\
\hline & \multicolumn{2}{|c|}{ North $N=14$} & \multicolumn{2}{|c|}{ Middle $\mathbf{N}=\mathbf{I}$ I } & \multicolumn{2}{|c|}{ South $\mathbf{N}=7$} & \multicolumn{2}{|c|}{ Total $\mathbf{N}=32$} \\
\hline & $\mathbf{n}$ & $\%$ & n & $\%$ & $\mathbf{n}$ & $\%$ & $\mathbf{n}$ & $\%$ \\
\hline Normal bed & 3 & 21.4 & 10 & 90.9 & 3 & 42.9 & 16 & 50.0 \\
\hline Transferable bed & 6 & 42.9 & 9 & 81.8 & 1 & 14.3 & 16 & 50.0 \\
\hline More than one delivery bed & 6 & 42.9 & 10 & 90.9 & 1 & 14.3 & 17 & 53.1 \\
\hline Obstetrical stethoscope & 9 & 64.3 & 10 & 90.9 & 2 & 28.6 & 21 & 65.6 \\
\hline Electronic thermometer for baby & 3 & 21.4 & 6 & 54.5 & 1 & 14.3 & 10 & 31.3 \\
\hline Ambu bag and mask for baby & 7 & 50.0 & 10 & 90.9 & 4 & 57.1 & 21 & 65.6 \\
\hline A radiant heater & 7 & 50.0 & 9 & 81.8 & 4 & 57.1 & 20 & 62.5 \\
\hline Adult blood pressure gauge & 9 & 64.3 & 11 & 100.0 & 4 & 57.1 & 24 & 75.0 \\
\hline Wall clock & 8 & 57.1 & 8 & 72.7 & 2 & 28.6 & 18 & 56.3 \\
\hline Sterile equipment for cutting and tying the cord & 13 & 92.9 & II & 100.0 & 6 & 85.7 & 30 & 93.8 \\
\hline Infant weighing scale & 7 & 50.0 & 9 & 81.8 & 2 & 28.6 & 18 & 56.3 \\
\hline Room thermometer & 4 & 28.6 & 4 & 36.4 & 2 & 28.6 & 10 & 31.3 \\
\hline Curtains on the window and on the door to insure privacy of the DR & 14 & 100.0 & 10 & 90.9 & 7 & 100.0 & 31 & 96.9 \\
\hline Water/towel/soap & 13 & 92.9 & II & 100.0 & 5 & 71.4 & 29 & 90.6 \\
\hline
\end{tabular}


in one hospital. Staff were seen washing hands between each baby in almost $53.6 \%$ of the hospitals.

Infants in NICUs were usually fed special formula in $26(83.9 \%)$ hospitals, breastfeeding in three hospitals, and expressed breast milk in two hospitals. The temperature was not recorded in the NICUs of 11 hospitals and the temperature was less than $25^{\circ} \mathrm{C}$ in the NICUs of 8 hospitals.

The number of hospitals with an adequate number of incubators was 23 (71.9\%), with an adequate number of CPAP machines was $10(31.3 \%)$, an adequate number of resuscitation tables was $23(71.9 \%)$, with an adequate number of lung ventilation devices was $22(68.8 \%)$, with an adequate number of pulse oximeters was 17 (53.1), and with an adequate number of phototherapy lamps was 16 (50.0\%). According to NICU staff, the NICUs of these hospitals needed 0-12 additional incubators, $0-3$ additional resuscitation tables, 0-8 additional CPAP machines, $0-5$ additional lung ventilation devices (ventilators), 0-25 additional pulse oximeters, and 0-10 additional phototherapy lamps.

\section{Other equipment, drugs, and supplies in the facility overall}

Availability and functionality of equipment, drugs, and supplies which provide basic maternal and newborn health care were checked in all selected hospitals. Many hospitals had insufficient supplies of inexpensive items such as essential equipment, consumables, and drugs (Table 4). Breast pumps, feeding cups, and hats were available in three hospitals only. A Bilirubin-meter and HIV testing kit were available in $18.8 \%$ of hospitals, surfactants in $40.6 \%$ of hospitals, chlorhexidine $7 \%$ for cord care in $28.1 \%$ of hospitals, TT vaccines in $31.3 \%$ of hospitals, and partograph in $56.3 \%$ of hospitals. The hospitals in the south were the least affected by the poor availability of such supplies whereas hospitals in the north were the most affected. Poor availability of the consumables and medications was more evident in the private sector followed by the $\mathrm{MoH}$. The university hospital had almost all necessary supplies and medications.

\section{Practices}

Table 5 shows the alignment of health care workers' practices with the WHO recommendations and guidelines. Based on interviews with health workers and direct observation, women are usually supported during labor in all selected hospitals. In specific, support provided to women during labor, initiation of breastfeeding and breastfeeding support/ counseling, postpartum care of the mother, mothers' counseling at discharge, dry and open cord care, management of

Table 4 The availability of other equipment, drugs and supplies in the hospital overall

\begin{tabular}{|c|c|c|c|c|c|c|c|c|}
\hline & \multicolumn{2}{|c|}{ North $N=14$} & \multicolumn{2}{|c|}{ Middle $\mathbf{N}=$ I I } & \multicolumn{2}{|c|}{ South $\mathbf{N}=7$} & \multicolumn{2}{|c|}{ Total $\mathbf{N}=32$} \\
\hline & $\mathbf{n}$ & $\%$ & $\mathbf{n}$ & $\%$ & $\mathbf{n}$ & $\%$ & $\mathbf{n}$ & $\%$ \\
\hline Towel for drying and wrapping babies & 9 & 64.3 & 8 & 72.7 & 5 & 71.4 & 22 & 68.8 \\
\hline Blood sugar sticks & II & 78.6 & 7 & 63.6 & 7 & 100.0 & 25 & 78.1 \\
\hline Breast pump & I & 7.1 & I & 9.1 & I & 14.3 & 3 & 9.4 \\
\hline Feeding cups & I & 7.1 & 0 & 0.0 & 2 & 28.6 & 3 & 9.4 \\
\hline Hats & 0 & 0.0 & 2 & 18.2 & I & 14.3 & 3 & 9.4 \\
\hline Oxygen supply/blender & 14 & 100.0 & 10 & 90.9 & 7 & 100.0 & 31 & 96.9 \\
\hline Bilirubinometer & 2 & 14.3 & 3 & 27.3 & I & 14.3 & 6 & 18.8 \\
\hline Eye cover & II & 78.6 & 9 & 81.8 & 5 & 71.4 & 25 & 78. I \\
\hline HIV testing kit & 2 & 14.3 & 3 & 27.3 & I & 14.3 & 6 & 18.8 \\
\hline Antibiotics (ampicillin, cefazolin, erythromycin, gentamicin or penicillin) & 14 & 100.0 & 9 & 81.8 & 7 & 100.0 & 30 & 93.8 \\
\hline IV fluids & 14 & 100.0 & 9 & 81.8 & 6 & 85.7 & 29 & 90.6 \\
\hline Corticosteroids & 14 & 100.0 & 7 & 63.6 & 6 & 85.7 & 27 & 84.4 \\
\hline Surfactants & 2 & 14.3 & 6 & 54.5 & 5 & 71.4 & 13 & 40.6 \\
\hline Aminophylline/caffeine & 14 & 100.0 & 7 & 63.6 & 7 & 100.0 & 28 & 87.5 \\
\hline Vitamin K (IM) & 14 & 100.0 & 7 & 63.6 & 7 & 100.0 & 28 & 87.5 \\
\hline Chlorexidhine seven for cord care & 2 & 14.3 & 5 & 45.5 & 2 & 28.6 & 9 & 28.1 \\
\hline Iron & 5 & 35.7 & 7 & 63.6 & 5 & 71.4 & 17 & 53.1 \\
\hline Folic acid & 6 & 42.9 & 7 & 63.6 & 5 & 71.4 & 18 & 56.3 \\
\hline TT vaccines & I & 7.1 & 5 & 45.5 & 4 & 57.1 & 10 & 31.3 \\
\hline Anithypertensive drugs & 13 & 92.9 & 7 & 63.6 & 7 & 100.0 & 27 & 84.4 \\
\hline Magnesium sulphate (injection) & 13 & 92.9 & 7 & 63.6 & 7 & 100.0 & 27 & 84.4 \\
\hline Partograph & 4 & 28.6 & 7 & 63.6 & 7 & 100.0 & 18 & 56.3 \\
\hline Uterotonic (oxytocin and/or misoprostol) & 12 & 85.7 & 7 & 63.6 & 6 & 85.7 & 25 & 78.1 \\
\hline Antibiotics (ampicillin) & 13 & 92.9 & 7 & 63.6 & 7 & 100.0 & 27 & 84.4 \\
\hline
\end{tabular}


Table 5 The alignment of practices with the WHO recommendations and guidelines

\begin{tabular}{|c|c|c|c|c|c|c|c|c|}
\hline \multirow[t]{3}{*}{ Practices } & \multicolumn{8}{|c|}{$\begin{array}{l}\text { Number of hospitals with practices that are in line with WHO } \\
\text { recommendations }\end{array}$} \\
\hline & \multicolumn{2}{|c|}{ North $N=14$} & \multicolumn{2}{|c|}{ Middle $\mathbf{N}=$ I I } & \multicolumn{2}{|c|}{ South $\mathbf{N}=7$} & \multicolumn{2}{|c|}{ Total $\mathbf{N}=32$} \\
\hline & $\mathbf{n}$ & $\%$ & $\mathbf{n}$ & $\%$ & $\mathbf{n}$ & $\%$ & $\mathbf{n}$ & $\%$ \\
\hline Support to the women during labor & 13 & 92.9 & II & 100.0 & 7 & 100.0 & 31 & 96.9 \\
\hline Free delivery position & 0 & 0.0 & 3 & 27.3 & $\mathrm{I}$ & 14.3 & 4 & 12.5 \\
\hline Use of partogram & 6 & 42.9 & II & 100.0 & 6 & 85.7 & 23 & 71.9 \\
\hline Skin-to-skin contact & 2 & 14.3 & 4 & 36.4 & 5 & 71.4 & $\mathrm{II}$ & 34.4 \\
\hline Transportation of the baby within maternity & II & 78.6 & 7 & 63.6 & 5 & 71.4 & 23 & 71.9 \\
\hline Initiation of breastfeeding and breastfeeding support/counseling & 13 & 92.9 & 10 & 90.9 & 6 & 85.7 & 29 & 90.6 \\
\hline Postpartum care of the mother & 13 & 92.9 & 11 & 100.0 & 5 & 71.4 & 29 & 90.6 \\
\hline Mother counseling at discharge & II & 78.6 & II & 100.0 & 6 & 85.7 & 28 & 87.5 \\
\hline Dry and open cord care & 12 & 85.7 & 10 & 90.9 & 7 & 100.0 & 29 & 90.6 \\
\hline Checking daily the healthy full-term baby weight & 1 & 7.1 & 7 & 63.6 & 3 & 42.9 & $\mathrm{II}$ & 34.4 \\
\hline Checking the baby temperature & 10 & 71.4 & 6 & 54.5 & 6 & 85.7 & 22 & 68.8 \\
\hline Assessing daily the correct attachment of the baby to the breast & 9 & 64.3 & 7 & 63.6 & 6 & 85.7 & 22 & 68.8 \\
\hline Management of asphyxia & 13 & 92.9 & II & 100.0 & 7 & 100.0 & 31 & 96.9 \\
\hline Management of jaundice & 13 & 92.9 & II & 100.0 & 7 & 100.0 & 31 & 96.9 \\
\hline
\end{tabular}

asphyxia, and management of jaundice were in line with the WHO recommendations in the majority of hospitals. However, some healthcare workers' practices in a few hospitals were not in line with these recommendations and guidelines. Interestingly, the practice of skin-to-skin contact (kangaroo care) and daily measurement of the healthy full-term baby weight were consistent with the WHO recommendations in about one-third of hospitals (34.4\%); however, free delivery position was practiced in only $12.5 \%$ of selected hospitals.

A total of $18(56.3 \%)$ hospitals had separate space (apart from the delivery room) for mothers and newborns together. Only two hospitals $(6.3 \%)$ practiced kangaroo mother care for all low birth weight babies (weight $<2,500$ grams) and $8(25.0 \%)$ hospitals practiced it for some low birth-weight babies.

\section{Documentation, guidelines, policies, and statistics}

Table 6 shows the availability of essential and important guidelines and policies. A large number of hospitals, mainly the hospitals in the north region, lacked important guidelines, protocols, and policies. Almost one-third (37.5\%) of hospitals lacked national guidelines for integrated management of pregnancy and childbirth (IMPAC), 46.9\% lacked protocols on referral of sick newborns, $37.5 \%$ lacked national guidelines for CEmOC, and 40.6\% lacked protocols on management of pre-term labor. Special policies for TB or HIV+ deliveries were present in 3 (9.4\%) hospitals only. Only $3(9.7 \%)$ hospitals participated in regular reviews of maternal or newborn deaths or "nearmisses" for both mothers and newborn. According to the health sector, almost all private hospitals lacked these guidelines/protocols.

\section{Staff training}

Interviews with the available health workers showed that there were gaps in the training of staff. Health care providers of delivery and newborn services in many hospitals did not receive any training or updates either on or off-site in some special skills. Training on neonatal resuscitation using bag and mask was provided for all staff in 14 hospitals only. None of the staff in 17 hospitals received training on the kangaroo care method for low birth-weight babies, and none of the staff in 20 hospitals received training on special delivery care practices for preventing mother-to-child transmission of HIV. A large number of health professionals in hospitals were not trained on essential and lifesaving skills and practices such as use of corticosteroids for preterm labor, sterile cord cutting and appropriate cord care, thermal care (including immediate drying and skin-to-skin care), newborn infection management (including injectable antibiotics), and breastfeeding (early and exclusive).

\section{Discussion}

The quality of maternal and child health facilities and services has been improving in Jordan; however, not all hospitals are providing a high level of quality to the same degree. There is significant variation in available resources between 
Table 6 The availability of essential and important guidelines, protocols and policies

\begin{tabular}{|c|c|c|c|c|c|c|c|c|}
\hline & \multicolumn{2}{|c|}{ North $N=14$} & \multicolumn{2}{|c|}{ Middle $\mathbf{N}=$ I I } & \multicolumn{2}{|c|}{ South $\mathbf{N}=7$} & \multicolumn{2}{|c|}{ Total $\mathbf{N}=32$} \\
\hline & $\mathbf{n}$ & $\%$ & $\mathbf{n}$ & $\%$ & $\mathbf{n}$ & $\%$ & $\mathbf{n}$ & $\%$ \\
\hline $\begin{array}{l}\text { National Guidelines for Integrated Management of Pregnancy and Childbirth } \\
\text { (IMPAC) available in this service site }\end{array}$ & 3 & 21.4 & 5 & 45.5 & 4 & 57.1 & 12 & 37.5 \\
\hline Guidelines or protocols on referral of sick newborns & 5 & 35.7 & 5 & 45.5 & 5 & 71.4 & 15 & 46.9 \\
\hline $\begin{array}{l}\text { National Guidelines for Comprehensive Emergency Obstetric Care } \\
\text { (CEmOC) }\end{array}$ & 3 & 21.4 & 5 & 45.5 & 4 & 57.1 & 12 & 37.5 \\
\hline Guidelines or protocols on management of pre-term labor & 4 & 28.6 & 5 & 45.5 & 4 & 57.1 & 13 & 40.6 \\
\hline $\begin{array}{l}\text { Register where client information from attended births is recorded, ie, a } \\
\text { delivery register }\end{array}$ & 8 & 57.1 & 8 & 72.7 & 6 & 85.7 & 22 & 68.8 \\
\hline Special policy for TB deliveries & 1 & 7.1 & I & 9.1 & I & 14.3 & 3 & 9.4 \\
\hline Special policy for HIV+ deliveries & 1 & 7.1 & I & 9.1 & I & 14.3 & 3 & 9.4 \\
\hline Relevant statistics and information (eg, admissions, outpatients, etc.) & 7 & 50.0 & 8 & 72.7 & 6 & 85.7 & 21 & 65.6 \\
\hline
\end{tabular}

geographical areas and the different health sectors in Jordan. This rapid assessment highlighted many deficiencies in providing high quality of care in some of the selected hospitals.

Our findings are in alignment with the WHO standards for provision of maternal and neonatal care (2016), ${ }^{3}$ stating that every woman and newborn should receive private care based on their needs and preferences in order to facilitate the continuity of care through having specific and maintained areas for labor, childbirth, and postnatal care. The WHO also encourages hospital managers to supervise the assessment and monitoring of the availability of resources and find out areas that need to be improved, thus promoting quality improvement.

Measures should be adapted to each context, but standards must be implemented consistently to ensure quality improvement. The quality measures include input, output/ process, and outcome measures. Input measures include physical resources, human resources, policies, protocols, and guidelines. Output measures include coverage of key practices. The outcome measures include people-centered and health outcomes.

Our findings with regard to human resources are alarming and are inconsistent with the WHO (2016) guidelines for providing optimal care for mothers and their newborns. In particular, standard 7 emphasizes having competent, motivated, and available staff to provide routine care and manage complications, but this is not the case in some hospitals in Jordan, according to the current project.

The assessed hospitals offer basic and comprehensive emergency care; however, these are not fully consistent with the recent 2016 WHO standards which state that routine care of newborns immediately after birth facilitates adaptation of the newborn to the new environment, meets the baby's immediate needs in the best possible way, and avoids preventable and avoidable complications. Any complications need to be correctly identified and managed carefully and in a comprehensive approach.

Other WHO quality statements emphasize routine assessment of women on admission and during labor to ensure essential care that is appropriate to the woman's case, to prevent the onset of complications and to identify risks or complications that require urgent action or referral for better outcomes of pregnancy and labor.

As noted with regard to antenatal care, some of the current findings do not fully adhere to the WHO guidelines of providing optimal care for mothers and newborns in the antenatal period, which stress the importance of effective prevention and management of conditions in late pregnancy in order to minimize the maternal death rate as well as antepartum-related stillbirths. On the basis of the current evidence on burden and impact, the following thematic areas are considered high priorities for evidence-based practices in routine and emergency care:

- management of preeclampsia, eclampsia, and its complications;

- management of preterm labor;

- management of maternal infections.

Our assessment demonstrated insufficient supplies of some highly imperative items in the admission departments of some hospitals, including, for example, an emergency set, which is lacking in three hospitals. Other important deficiencies that were seen in 5-6 hospitals included magnesium sulphate, diazepam, and oxytocin. Magnesium sulphate, for instant, is an antihypertensive medication, which is used to prevent hypertension-related complications for women with preeclampsia. In its quality statement number 1.2 , the 
WHO (2016) recommended that women with preeclampsia or eclampsia should promptly receive appropriate interventions. Therefore, magnesium sulphate, along with the other medications, should be prepared beforehand to be ready for any potential crisis that may put mother and infant at risk during labor and delivery. ${ }^{20}$

This assessment indicated that the operation theaters in many hospitals lacked a variety of necessary equipment, including some basic items such as thermometers and some advanced items such as advanced resuscitation sets for babies and a transportation heated cradle/incubator. The WHO stressed the need for providing a transportation incubator for each delivery room, to be used when the newborn is transferred to another area. ${ }^{21}$ OTs are active and complex environments which require great attention in order to ensure highest level of patient safety. Harm to mothers and newborn babies may occur due to the lack of necessary equipment and supplies. OTs should provide basic and advance resuscitation for newborn babies. The WHO $(2016)^{3}$ recommended in its quality statement \# 1.5 that newborns who are not breathing spontaneously after birth should receive resuscitation with a bag and mask within 1 minute of birth. In low resource settings and whenever health care staff cannot handle complications that may arise during labor, immediate referral for both mother and infant should be available.

It is recommended that every delivery room should be a safe environment for both mothers and newborn babies. Only two-thirds of all delivery rooms in the selected hospitals had radiant heaters and obstetrical stethoscopes available. Delivery rooms should have an appropriate physical environment and adequate supplies for both routine and advanced management of complications, including emergency resuscitation equipment that is available and ready to use. . $^{3,22}$

The assessment revealed a marked geographical variation in relation to available NICU equipment. Hospitals located in the South of Jordan had better scores in the number of incubators in their NICUs than hospitals in the North and the Middle. Meanwhile, hospitals in the North scored better than the rest of the country's hospitals in relation to the availability of resuscitation tables, $\mathrm{CPAP}, \mathrm{O}_{2}$ oximeters, and phototherapy. Generally, all hospitals need to increase their capacity of this equipment. One-third of all assessed hospitals did not have resuscitation sets in all sections. It has been reported that almost $25 \%$ of first week neonatal deaths can be prevented by immediate basic resuscitation. ${ }^{23}$ Availability of essential resuscitation equipment and well trained staff is likely to reduce intra-partum-related neonatal deaths by $30 \% .{ }^{24}$
Although a breastfeeding room was available in all hospitals except four, the majority of infants $(83.9 \%)$ received infant formula, while only $9.7 \%$ of all hospitals offered breastfeeding, with two hospitals $(6.5 \%)$ providing expressed breast milk. Breastfeeding and providing newborn babies with expressed mother's milk was proved to have many benefits to a child's health and therefore should be facilitated and encouraged.

As noticed in our results regarding the availability of equipment, drugs, and supplies, a significant lack of necessary equipment, drugs, and supplies was evident. This is in contrast to the WHO standards for improving quality of maternal and newborn care in health facilities (2016), in particular standard 8, which emphasize the provision of optimal quality care through ensuring available, adequate essential medicines, equipment, and other supplies.

Furthermore, the WHO (2016) stressed the importance of the availability of a list of important medicines and supplies and a secure storage, with a good system to prevent lack of these items. For example, one quality statement in standard eight ensures that all suitable medicines are available in the relevant maternal wards and departments whereas necessary equipment is available in the right places, at all times, with a system for constant maintenance and guidelines for correct equipment usage. Unfortunately, these WHO quality statements are not fully reflected in several Jordanian hospitals.

While our findings of the practices align with standard 1 of the WHO guidelines and quality statements related to providing optimal maternal and newborn care, the practices for routine care and management of complications during labor, childbirth, and the early postnatal period were not totally evidence-based. Providing up-to-date evidence-based practices, according to the WHO recommendations, can significantly decrease the number of deaths and improve outcomes of women and newborns during labor and the early postnatal period.

Our findings relating to documentation, guidelines, policies, and statistics are disappointing and imply poor maternal and newborn care in Jordan due to the lack of guidelines and protocols in several hospitals. One of the WHO (2016) standards of care (standard 1) has nine quality statements, which include how to care for mothers/newborns in emergency or critical cases. If such guidelines are not available in hospitals, poor maternal and newborn outcomes, and hence high rates of morbidity and mortality, are expected.

As found in our study, there is a huge lack of healthcare professionals training on routine and special care for mothers and newborns, including emergency and critical care. This 
is in contrast to the WHO (2016) guidelines for the provision of maternal and newborn care, particularly standard 7 , which focuses on having skilled, competent birth attendants and support staff to meet requirements during labor, childbirth, and the early postnatal period and thus improve their performance and roles.

In order to accomplish this standard, the WHO recommends that all healthcare providers need to have a job description that outlines the required competence, roles, and responsibilities, supported by the desired qualifications. This can be achieved through special programs for recruitment, retention, professional development, and higher education for all healthcare providers.

It is essential to provide whatever is needed for a safe and healthy delivery to guarantee a high quality of care for both mother and newborn. Identification of deficiencies in maternity hospitals is important for further improvement, and therefore, substantial reduction in mortality rates among these vulnerable populations.

This study has many strengths that need to be acknowledged. The study was multisite and multi-sector since it included hospitals from 12 different governorates in Jordan and from all health sectors, thus increasing generalizability of the findings on the national level. Additionally, we assessed quality of care of maternal and newborn care using a comprehensive, validated checklist that reflected all the WHO standards for easier and more accurate comparisons (various services for mothers and newborns, availability of resources, equipment and supplies, documentation and staff training, and provision of health care services). Importantly, we used the triangulation method during data collection to improve the credibility and reliability of findings. In particular, the assessment forms utilized different sources of information from hospitals during data collection, including direct observation of cases, and semi-structured interviews with staff. Finally, the sample size was large, resulting in a representative sample.

Our results are presented according to the region. However, the geographical differences in the studied parameters might not reflect true geographical differences. The apparent differences in the studied parameters might reflect the differences between different sectors. Therefore, one should be cautious in the interpretations of the study findings.

\section{Conclusion}

This study showed that, overall, the quality of the provision of maternal and newborn care in many Jordanian hospitals from different governorates and health sectors was adequate. Nonetheless, some deficiencies were found when compared with the WHO (2016) guidelines for optimal maternal and newborn care across some of the recruited hospitals. Some of these deficiencies included shortages of skilled and competent birth attendants, lack of optimal thorough antenatal care, and lack of necessary supplies, drugs, equipment, and resources during labor and the early postnatal care. Importantly, we found that some healthcare professionals are not regularly trained in routine and special care needed for labor and the early postpartum period in both normal and complicated births. Finally, our rapid assessment of the maternal and newborn services in Jordanian hospitals revealed the lack of pivotal protocols, policies, and guidelines necessary for optimal care. Improvement of the quality of preventive and curative care during this critical period could improve the survival rate of the mother and her newborn. Assessing the current status of the quality of care in providing maternal and newborn health care according to recognized quality standards can pinpoint the areas that need to be improved to reach optimal care for both the mother and her fetus/newborn. The deficiencies revealed from this assessment can be utilized by health professionals and stakeholders to provide optimal quality care for women and their newborns. One way is to enforce health programs to develop and implement health services to close the gap between the current situation and that recommended by the WHO.

\section{Acknowledgment}

The authors would like to acknowledge Koninklijk Instituut voor de Tropen (Share-Net International) for their financial and technical support.

\section{Disclosure}

The authors report no conflicts of interest in this work.

\section{References}

1. Buttell P, Hendler R, Daley J. Quality in healthcare: concepts and practice. In: The Business of HealthCare: Improving Systems of Care. Westport, CT: Praeger; 2008.

2. Hulton L, Matthews Z, Stones RW. A Framework for the Evaluation of Quality of Care in Maternity Services. UK: University of Southampton; 2000.

3. World Health Organization [webpage on the Internet]. Standards for improving quality of maternal and newborn care in health facilities; 2016. Available from: http://www.who.int/maternal_child_adolescent/ documents/improving-maternal-newborn-care-quality/en/. Accessed March 13, 2018.

4. World Health Organization [webpage on the Internet]. Maternal deaths fell 44\% since 1990 - UN; 2016. Available from: http://www.who.int/ mediacentre/news/releases/2015/maternal-mortality/en/. Accessed March 13, 2018.

5. Ministry of Health. Essential Obstetric Care Clinical Guidelines for Physicians, H.S. strengthening. Amman, Jordan: 2007.

6. Amarin Z, Khader Y, Okour A, Jaddou H, Al-Qutob R. National maternal mortality ratio for Jordan, 2007-2008. Int J Gynaecol Obstet. 2010;111(2):152-156. 
7. Department of Statistics and ICF International. Jordan Population and Family Health Survey 2012. Calverton, Maryland, USA: Department of Statistics and ICF International; 2013.

8. Ministry of Health, Annual Statistical Report 2007 [webpage on the Internet]. Ministry of Health, Amman, Jordan; 2008. Available from: http:/ apps.moh.gov.jo/MOH/En/publications.php. Accessed March 13, 2018.

9. Dababneh F, Gerber DR, As'ad DM. Mortality in Jordan 2005. Directorate of Information Studies and Research. 2008, Ministry of Health, Amman. Jordan. 2005.

10. World Health Organization [webpage on the Internet]. Neonatal and Perinatal Mortality: Country, Regional and Global Estimates. 2006, World Health Organization: Geneva, Switzerland. Available from: http:// apps.who.int/iris/handle/10665/43444. Accessed March 13, 2018.

11. United Nations Sustainable Development [webpage on the Internet] Health - United Nations Sustainable Development; 2015. Available from: http://www.un.org/sustainabledevelopment/health/\#. Accessed March 13, 2018.

12. WHO. UNICEF. 2014. Every Newborn: An Action Plan to End Preventable Deaths. Geneva: World Health Organization.

13. Batieha AM, KhaderYS, Berdzuli N, et al. Level, causes and risk factors of neonatal mortality, in Jordan: results of a national prospective study. Matern Child Health J. 2016;20(5):1061-1071.

14. Raven JH, Tolhurst RJ. Tang S, van den Broek N. What is quality in maternal and neonatal health care? Midwifery. 2012;28(5):e676-e683.

15. Campbell OMR, Graham WJ; Lancet Maternal Survival Series Steering Group. Strategies for reducing maternal mortality: getting on with what works. Lancet. 2006;368(9543):1284-1299.

16. Global Health Sciences. Where Women Go to Deliver: Overview of the Project and Review of Preliminary Findings. University of California at San Francisco: San Francisco, California; 2014.
17. Bohren MA, Hunter EC, Munthe-Kaas HM, Souza JP, Vogel JP, Gülmezoglu AM. Facilitators and barriers to facility-based delivery in low- and middle-income countries: a qualitative evidence synthesis. Reprod Health. 2014;11(1):71.

18. The DHS Program Demographic and Health Surveys [webpage on the Internet]. ICF International. The Service Provision Assessment (SPA); 2017. Available form: https://dhsprogram.com/What-We-Do/SurveyTypes/SPA.cfm. Accessed March 13, 2018.

19. Healthy Newborn Network (HNN) and Inter-agency Newborn Indicators Technical Working Group. Newborn Services Rapid Health Facility Assessment tool. 2012. Available form: http://www.healthynewbornnetwork.org/resource/newborn-services-rapid-health-facility-assessment. Accessed March 13, 2018.

20. World Health Organization. WHO Safe Childbirth Checklist Implementation Guide: Improving the Quality of Facility-Based Delivery for Mothers and Newborns: Geneva, Switzerland: 2015.

21. World Health Organization. Essential Antenatal, Perinatal and Postpartum Care. Training Module. Regional Office for Europe, 2002;276-288. Available form: http://www.euro.who.int/_data/assets/ pdf_file/0013/131521/E79235.pdf. Accessed March 13, 2018.

22. Royal College of Obstetricians and Gynaecologists. Safer Childbirth: Minimum Standards for the Organisation and Delivery of Care in Labour. London: RCOG Press; 2007.

23. Wall SN, Lee AC, Carlo W, et al. Reducing intrapartum-related neonatal deaths in low- and middle-income countries - what works? Semin Perinatol. 2010;34(6):395-407.

24. Lee AC, Cousens S, Wall SN, et al. Neonatal resuscitation and immediate newborn assessment and stimulation for the prevention of neonatal deaths: a systematic review, meta-analysis and Delphi estimation of mortality effect. BMC Public Health. 2011;11(Suppl 3):S12. 


\section{Supplementary materials}

\section{Supplementary material S1}

Maternal and Newborn Services

Rapid Facility Assessment Forms

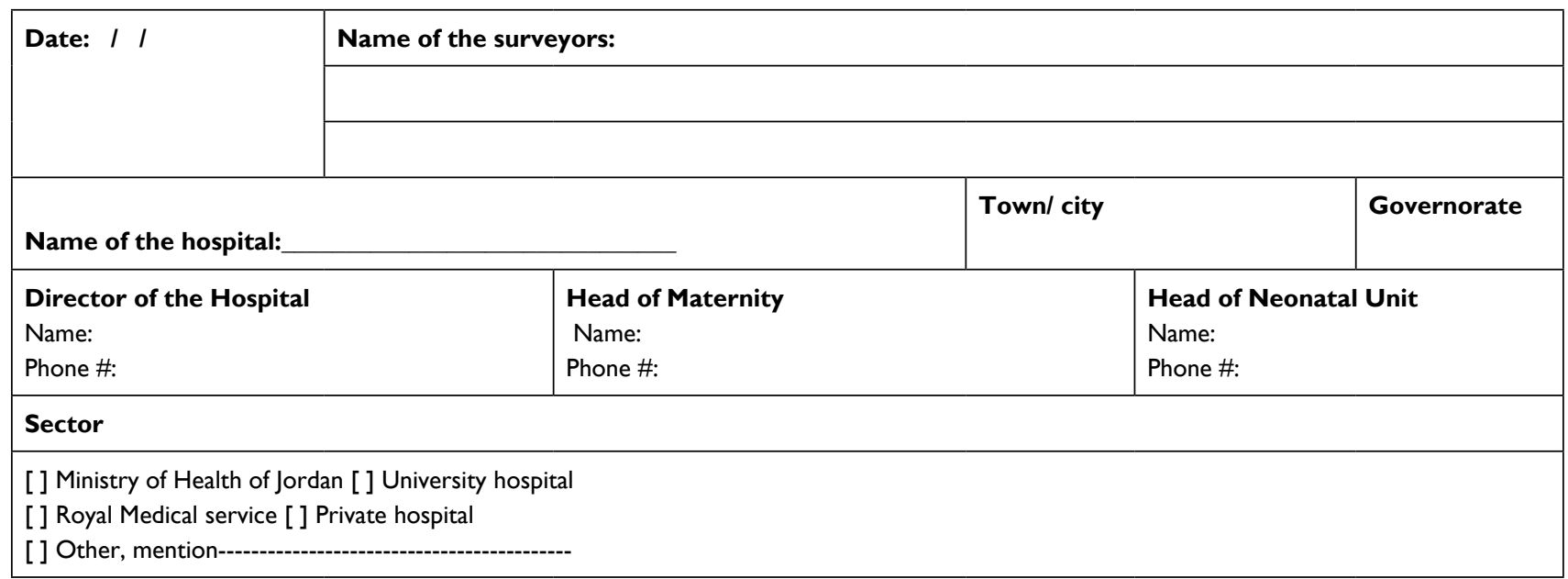

\section{A. Service availability}

[Please interview both the head of the maternity and the head of neonatal unit and ask about the availability of the following services]

\begin{tabular}{|c|c|c|c|c|}
\hline \multicolumn{2}{|c|}{ Availability of services } & \multirow[b]{2}{*}{ I. Yes } & \multirow[b]{2}{*}{ 2. No } & \multirow{2}{*}{$\begin{array}{l}\text { If yes, how } \\
\text { many beds? }\end{array}$} \\
\hline I & Does the health facility have a ward for admitting obstetrics patients? & & & \\
\hline 2 & Does the health facility have a theatre to perform CS? & I. Yes & 2. No & \\
\hline 3 & $\begin{array}{l}\text { Does the health facility have a separate room or ward for admitting "infectious" cases } \\
\text { (isolation)? }\end{array}$ & I. Yes & 2. No & \\
\hline 4 & $\begin{array}{l}\text { Does the health facility have a separate ward or room for admitting newborn babies } \\
\text { (nursery)? }\end{array}$ & I. Yes & 2. No & \\
\hline 5 & Does the health facility have a neonatal intensive care unit? & I. Yes & 2. No & \\
\hline
\end{tabular}

\section{B. Human resources}

B.1 Is a person (specialist/resident) skilled in conducting deliveries present at the facility or on call at all times (24 hours a day), including weekends, to provide delivery care?
1. Yes, present
2. Yes, on call
3. No 24-hour coverage

\begin{tabular}{|l|l|l|l|l|l|}
\hline B.2 Number of permanent staff providing perinatal care in the facility & \multicolumn{2}{l|}{$\begin{array}{l}\text { If inadequate, how } \\
\text { many more is } \\
\text { needed? }\end{array}$} \\
\hline & & Number & Adequacy of the staff & 3.Inadequate & \\
\hline I. Ob\&Gyns & & & & & \\
\hline 2. Pediatricians & & & & & \\
\hline 3. Neonatologists & & & & & \\
\hline 4. Residents in Ob\&Gyns & & & & & \\
\hline 5. Residents in Pediatrics & & & & & \\
\hline 6. Midwives/nurses (obstetrics) & & & & & \\
\hline 7. Pediatric Nurses & & & & & \\
\hline
\end{tabular}




\section{Basic and comprehensive emergency obstetric care and neonatal care}

\begin{tabular}{|l|l|l|l|l|l|}
\hline \multicolumn{2}{|l|}{ Please tell me if the facility is able to provide any of the following services and if it is currently provided. } \\
\hline Type of service & \multicolumn{2}{l|}{ Able to provide it } & \multicolumn{2}{l|}{ Currently provides it } \\
\hline I. & Normal vaginal delivery & I. Yes & 2. No & I. Yes & 2. No \\
\hline 2. & Cesarean section & I. Yes & 2. No & I. Yes & 2. No \\
\hline 3. & Administration of parenteral antibiotics; (IV or IM) & I. Yes & 2. No & I. Yes & 2. No \\
\hline 4. & Administration of uterotonic drugs (ie, parenteral oxytocin); (IV OR IM) & I. Yes & 2. No & I. Yes & 2. No \\
\hline 5. & $\begin{array}{l}\text { Administration of parenteral anticonvulsants for preeclampsia and eclampsia } \\
\text { (ie, magnesium sulphate) }\end{array}$ & I. Yes & 2. No & I. Yes & 2. No \\
\hline 6. & Manual removal of the placenta & I. Yes & 2. No & I. Yes & 2. No \\
\hline 7. & Removal of retained products after delivery (eg, manual vacuum aspiration) & I. Yes & 2. No & I. Yes & 2. No \\
\hline 8. & Perform assisted vaginal delivery (eg vacuum extraction, forceps delivery) & I. Yes & 2. No & I. Yes & 2. No \\
\hline 9. & Perform blood transfusion & I. Yes & 2. No & I. Yes & 2. No \\
\hline 10. & Perform basic neonatal resuscitation (eg, with bag and mask) & I. Yes & 2. No & I. Yes & 2. No \\
\hline II. & Corticosteroids for preterm labour & I. Yes & 2. No & I. Yes & 2. No \\
\hline I2. & Non-invasive ventilation (CPAP) for baby & I. Yes & 2. No & I. Yes & 2. No \\
\hline 13. & Intubation/ventilation for baby & I. Yes & 2. No & I. Yes & 2. No \\
\hline
\end{tabular}

\section{Antenatal care}

[Ask the head of the maternity]

\section{D1. Does this facility provide antenatal care?}

1. Yes [if yes fill in the table below] 2. No [If no go to section E]

\begin{tabular}{|l|l|l|l|l|}
\hline \multicolumn{2}{|l|}{ D2. If the facility provides antenatal care, are the following services systematically provided during pregnancy? } \\
\hline \multicolumn{2}{|l|}{ Service } & $\begin{array}{l}\text { YES systematically } \\
\text { provided }\end{array}$ & $\begin{array}{l}\text { YES but not } \\
\text { systematically }\end{array}$ & NOT provided \\
\hline I. & Iron supplementation & I & 2 & 3 \\
\hline 2. & Folic acid supplementation & I & 2 & 3 \\
\hline 3. & Fasting blood sugar & I & 2 & 3 \\
\hline 4. & Blood pressure & I & 2 & 3 \\
\hline 5. & Hemoglobin & I & 2 & 3 \\
\hline 6. & Urine stick (protein, sugar) & I & 2 & 3 \\
\hline 7. & Tetanus toxoid (TT) vaccination & I & 2 & 3 \\
\hline 8. & Breastfeeding counseling & I & 2 & 3 \\
\hline 9. & Family planning counseling & I & 2 & 3 \\
\hline
\end{tabular}

\section{E. Admission department (maternity)}

Please observe and indicate if the following are available in the admission department:

1. Blood pressure gauge

2. Obstetrical stethoscope

3. Stethoscope for adults

4. Thermometer

5. Couch

6. Soap

7. Towels

8. Posters or information on hand washing
1. Yes [ ] 2. No [ ]

1. Yes [ ] 2. No [ ]

1. Yes [ ] 2. No [ ]

1. Yes [ ] 2. No [ ]

1. Yes [ ] 2. No [ ]

1. Yes [ ] 2. No [ ]

1. Yes [ ] 2. No [ ]

1. Yes [ ] 2. No [ ] 
9. Surgical transport like wheel chair

10. Emergency set:

10.1 Bag and mask for adults

10.2 IV line

10.3 Syringes

10.4 IV catheter

10.5 Sterile medical gloves

10.6 Normal saline

10.7 Magnesium sulfate

10.8 Diazepam

10.9 Oxytocin
1. Yes [ ] 2. No [ ]

1. Yes [ ] 2. No [ ]

1. Yes [ ] 2. No [ ]

1. Yes [ ] 2. No []

1. Yes [ ] 2. No [ ]

1. Yes [ ] 2. No [ ]

1. Yes [ ] 2. No [ ]

1. Yes [ ] 2. No [ ]

1. Yes [ ] 2. No [ ]

1. Yes [ ] 2. No []

\section{F. Delivery room}

\section{F.1 Number of delivery rooms in the facility [ ]}

\begin{tabular}{|c|c|c|c|c|}
\hline \multicolumn{5}{|c|}{ F.2 Please observe and indicate if the following are available in the delivery room/s: } \\
\hline \multicolumn{2}{|c|}{ Safety/Saving life medical equipment } & \multicolumn{3}{|l|}{ Availability } \\
\hline & & \multirow[t]{2}{*}{$\begin{array}{l}\text { Available in all } \\
\text { delivery rooms }\end{array}$} & \multirow[t]{2}{*}{$\begin{array}{l}\text { Available in some } \\
\text { delivery rooms }\end{array}$} & \multirow[t]{2}{*}{ Not available } \\
\hline I. & Normal bed & & & \\
\hline 2. & Transferable bed & & & \\
\hline 3. & More than one delivery bed & & & \\
\hline 4. & Obstetrical stethoscope & & & \\
\hline 5. & Electronic thermometer for baby & & & \\
\hline 6. & Ambu bag and mask for baby & & & \\
\hline 7. & A radiant heater & & & \\
\hline 8. & Adult blood pressure gauge & & & \\
\hline 9. & Wall clock & & & \\
\hline 10. & Sterile equipment for cutting and tying the cord & & & \\
\hline 11. & Infant weighing scale & & & \\
\hline 12. & Room thermometer & & & \\
\hline 13. & Curtains on the window and on the door to insure privacy of the DR & & & \\
\hline 14. & Water/towel/soap & & & \\
\hline
\end{tabular}

\section{G. Delivery block/department}

\begin{tabular}{|c|c|c|c|}
\hline \multicolumn{4}{|c|}{ Please observe and indicate if the following are available in the delivery block/department. } \\
\hline I. & Is there an emergency set for advanced newborn resuscitation? & I. Yes & 2. No \\
\hline 2. & Laryngoscope (in work condition) & I. Yes & 2. No \\
\hline 3. & Blades of 2 sizes & I. Yes & 2. No \\
\hline 4. & Intubation's tubes (4 sizes) & I. Yes & 2. No \\
\hline 5. & T-adapter & I. Yes & 2. No \\
\hline 6. & Aspiration catheter & I. Yes & 2. No \\
\hline 7. & Adrenalin & I. Yes & 2. No \\
\hline 8. & Syringes $(5 \mathrm{ml})$ & I. Yes & 2. No \\
\hline 9. & Normal saline & I. Yes & 2. No \\
\hline 10. & Sterile gloves & I. Yes & 2. No \\
\hline II. & How many emergency sets are in delivery block/department?* & & \\
\hline 12. & Is there a separate fridge for blood components? & I. Yes & 2. No \\
\hline
\end{tabular}




\section{H. Operation theater (OT)}

\section{H1. Number of operation theaters I \\ H2. Check in each operation theater}

\begin{tabular}{|c|c|c|c|c|}
\hline \multicolumn{2}{|c|}{ Safety/saving life medical equipment } & \multirow{2}{*}{$\begin{array}{l}\text { Available in } \\
\text { all operation } \\
\text { theaters }\end{array}$} & \multirow{2}{*}{$\begin{array}{l}\text { Available in } \\
\text { some operation } \\
\text { theaters }\end{array}$} & \multirow[t]{2}{*}{ Not available } \\
\hline 4.1 & Is there a thermometer? & & & \\
\hline 4.3 & Are there Ambu bags and masks for baby? & & & \\
\hline 4.4 & Is there a set of advanced resuscitation for the baby? & & & \\
\hline 4.5 & $\begin{array}{l}\text { Is there a warming device for the newborn in the operation theater (radiant } \\
\text { heater, incubator)? }\end{array}$ & & & \\
\hline 4.6 & Is there a wall clock with a second hand in the operation theater? & & & \\
\hline 4.7 & Is there a transportation (portable) incubator for baby in the operation theater? & & & \\
\hline 4.8 & Is there an adult blood pressure gauge? & & & \\
\hline 4.9 & Is oxygen available? & & & \\
\hline
\end{tabular}

\section{NICU}

What is the maximum capacity of infants in the NICU? [__ ]

What is the total number of nurses working in NICU? [_ $]$

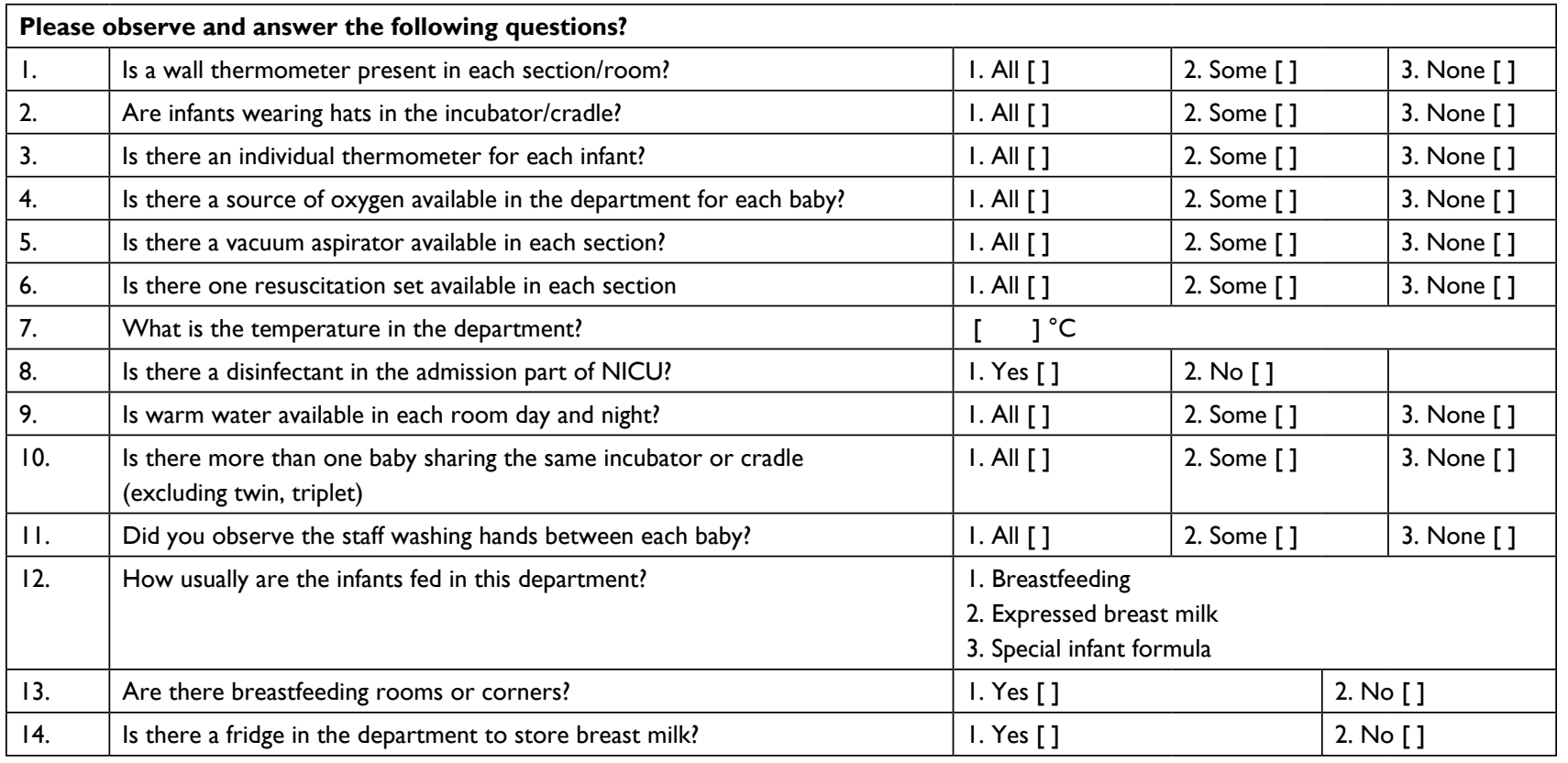

\begin{tabular}{|c|c|c|c|c|}
\hline \multicolumn{5}{|c|}{ Please interview the neonatologist and ask him/her these questions. } \\
\hline \multicolumn{2}{|c|}{ Equipment } & \multirow{2}{*}{$\begin{array}{l}\text { How many in the } \\
\text { department? }\end{array}$} & \multirow{2}{*}{$\begin{array}{l}\text { Are they sufficient } \\
\text { I. Yes [ ] 2. No [ ] }\end{array}$} & \multirow{2}{*}{$\begin{array}{l}\text { How many more are } \\
\text { needed if not sufficient? }\end{array}$} \\
\hline I. & Incubators & & & \\
\hline 2. & Resuscitation table & & I. Yes [] 2. No [ ] & \\
\hline 3. & Continuous positive airway pressure (CPAPs) & & I. Yes [] 2. No [ ] & \\
\hline 4. & Lung ventilation devices (ventilators) & & I. Yes [ ] 2. No [ ] & \\
\hline 5. & Pulse oxymeters & & I. Yes [] 2. No [ ] & \\
\hline 6. & Phototherapy lamps & & I. Yes [] 2. No [ ] & \\
\hline
\end{tabular}


J. Other equipment, drugs and supplies in the facility overall

\begin{tabular}{|c|c|c|c|c|}
\hline \multicolumn{5}{|c|}{ I would like to know if the following items are available in the facility (if not functional then it is unavailable) } \\
\hline & & \multicolumn{3}{|l|}{ Availability } \\
\hline & & $\begin{array}{l}\text { Reported and } \\
\text { observed }\end{array}$ & $\begin{array}{l}\text { Reported and } \\
\text { not observed }\end{array}$ & Not available \\
\hline I. & Towel for drying and wrapping babies & I & 2 & 3 \\
\hline 2. & Blood sugar sticks & $\mathrm{I}$ & 2 & 3 \\
\hline 3. & Breast pump & 1 & 2 & 3 \\
\hline 4. & Feeding cups & $\mathrm{I}$ & 2 & 3 \\
\hline 5. & Hat & I & 2 & 3 \\
\hline 6. & Oxygen supply/blender & $\mathrm{I}$ & 2 & 3 \\
\hline 7. & Bilirubinometer & I & 2 & 3 \\
\hline 8. & Eye cover & $\mathrm{I}$ & 2 & 3 \\
\hline 9. & HIV testing kit & 1 & 2 & 3 \\
\hline 10. & Antibiotics (ampicillin, cefazolin, erythromycin, gentamicin, or penicillin) & 1 & 2 & 3 \\
\hline 11. & IV fluids & 1 & 2 & 3 \\
\hline 12. & Corticosteroids & 1 & 2 & 3 \\
\hline 13. & Surfactants & 1 & 2 & 3 \\
\hline 14. & Aminophylline/caffeine & 1 & 2 & 3 \\
\hline 15. & Vitamin K (IM) & 1 & 2 & 3 \\
\hline 16. & Chlorexidhine $7 \%$ for cord care & 1 & 2 & 3 \\
\hline 17. & Iron & 1 & 2 & 3 \\
\hline 18. & Folic acid & 1 & 2 & 3 \\
\hline 19. & TT vaccines & 1 & 2 & 3 \\
\hline 20. & Anithypertensive drugs & 1 & 2 & 3 \\
\hline 21. & Magnesium sulphate (injection) & 1 & 2 & 3 \\
\hline 22. & Partograph & 1 & 2 & 3 \\
\hline 23. & Uterotonic (oxytocin and/or misoprostol) & 1 & 2 & 3 \\
\hline 24. & Antibiotics (ampicillin) & 1 & 2 & 3 \\
\hline
\end{tabular}

\section{K. Practices}

Based on the interview with available health care providers, which of the following are implemented according to WHO recommendations?

\begin{tabular}{|l|l|l|}
\hline & \multicolumn{2}{|l|}{ In line with WHO recommendation } \\
\hline Topic & Yes & No \\
\hline I. Support to the women during labor & & \\
\hline 2. Free delivery position & & \\
\hline 3. Use of partogram & & \\
\hline 4. “Skin-to-skin" contact & & \\
\hline 5. Transportation of the baby within maternity & & \\
\hline 6. Initiation of breastfeeding and breastfeeding support/counseling & & \\
\hline 7. Postpartum care of the mother & & \\
\hline 8. Mother counseling at discharge & & \\
\hline 9. Dry and open cord care & & \\
\hline 10. Checking daily the healthy full-term baby weight & & \\
\hline II. Checking the baby temperature & & \\
\hline I2. Assessing daily the correct attachment of the baby to the breast & & \\
\hline 13. Management of asphyxia & & \\
\hline 14. Management of jaundice & & \\
\hline
\end{tabular}


Does the facility have a separate space (apart from the delivery room) for mothers and newborns together or for newborns only (postnatal area/ward)?

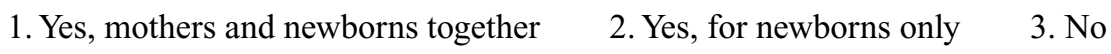

Does this facility practice Kangaroo Mother Care for low birth-weight babies?

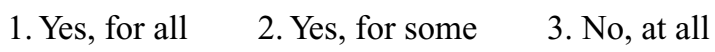

\section{Documentation, guidelines, policies, and statistics}

\begin{tabular}{|c|c|c|c|c|}
\hline \multicolumn{5}{|c|}{ Please interview both the head of the maternity and the head of neonatal unit and ask the following questions } \\
\hline & & $\begin{array}{l}\text { Yes, } \\
\text { observed }\end{array}$ & $\begin{array}{l}\text { Yes, not } \\
\text { seen }\end{array}$ & $\begin{array}{l}\text { Not } \\
\text { available }\end{array}$ \\
\hline I. & $\begin{array}{l}\text { Do you have the national guidelines for Integrated Management of Pregnancy and } \\
\text { Childbirth (IMPAC) available in this service site? }\end{array}$ & & & \\
\hline 2. & Do you have guidelines or protocols on referral of sick newborns? & & & \\
\hline 3. & $\begin{array}{l}\text { Do you have the national guidelines for Comprehensive Emergency Obstetric Care } \\
\text { (CEmOC)? }\end{array}$ & & & \\
\hline 4. & Do you have guidelines or protocols on management of preterm labor? & & & \\
\hline 5. & $\begin{array}{l}\text { Is there a register where client information from attended births is recorded, ie, a } \\
\text { delivery register? }\end{array}$ & & & \\
\hline 6. & Does this facility have a special policy for TB deliveries? & & & \\
\hline 7. & Does this facility have a special policy for HIV+ deliveries? & & & \\
\hline 8. & Do you have relevant statistics and information (eg, admissions, outpatients, etc.)? & & & \\
\hline 9. & \multicolumn{4}{|c|}{$\begin{array}{l}\text { Does the facility participate in regular reviews of maternal or newborn deaths or "near-misses"? } \\
\text { I. Yes for mothers only 2. Yes for newborns only } \\
\text { 3. Yes for both mothers and newborn } 4 \text {. No doesn't participate }\end{array}$} \\
\hline
\end{tabular}

\section{Trained staff}

Please ask the following questions to all available providers of delivery and newborn services

\begin{tabular}{|l|l|l|l|l|}
\hline \multicolumn{2}{|l|}{ Have you received any training or updates either on or off-site in any of the following topics within the past 2 years? } \\
\hline & $\begin{array}{l}\text { Yes, all } \\
\text { staff }\end{array}$ & $\begin{array}{l}\text { Yes, most } \\
\text { of them }\end{array}$ & $\begin{array}{l}\text { Yes, some } \\
\text { of them }\end{array}$ & $\begin{array}{l}\text { None of } \\
\text { them }\end{array}$ \\
\hline & Neonatal resuscitation using bag and mask & & & \\
\hline & Breastfeeding (early and exclusive) & & & \\
\hline & Newborn infection management (including injectable antibiotics) & & & \\
\hline & Thermal care (including immediate drying and skin-to-skin care) & & & \\
\hline & Sterile cord cutting and appropriate cord care & & & \\
\hline & Kangaroo method for low birth-weight babies & & & \\
\hline $\begin{array}{l}\text { Special delivery care practices for preventing mother-to-child transmission of } \\
\text { HIV }\end{array}$ & & & & \\
\hline & Use of corticosteroids for preterm labor & & & \\
\hline
\end{tabular}




\section{Publish your work in this journal}

The Journal of Multidisciplinary Healthcare is an international, peerreviewed open-access journal that aims to represent and publish research in healthcare areas delivered by practitioners of different disciplines. This includes studies and reviews conducted by multidisciplinary teams as well as research which evaluates the results or conduct of such teams or health care processes in general. The journal covers a very wide range of areas and welcomes submissions from practitioners at all levels, from all over the world. The manuscript management system is completely online and includes a very quick and fair peer-review system. Visit http://www.dovepress.com/ testimonials.php to read real quotes from published authors. 